\title{
Leader Following With Non-Homogeneous Weights for Control of Vehicle Formations
}

\author{
Andrés A. Peters ${ }^{1}$, Oliver Mason ${ }^{2}$ and Richard H. Middleton ${ }^{3}$
}

\begin{abstract}
In this paper, we study a formation control scheme that achieves a tight formation in a 1D platoon. The scheme achieves constant inter-vehicle spacings (with no transient) for almost every vehicle pair whenever there are no disturbances. We build up from the basic leader following approach with a modification in the weight selection. In particular, each member tracks the movement of its immediate predecessor but also uses the leader state, which needs to be transmitted, in order to achieve a tight formation. The key design choice is the use of filters for the measurements that set the transfer functions from the leader trajectory to the inter-vehicle spacings to zero whenever possible. We support the analysis of the architecture with numerical simulations.
\end{abstract}

\section{INTRODUCTION}

Formation control of autonomous vehicles is a topic that has received an important amount of attention in recent decades. [1], [2], [3], [4]. Its importance in applications to automated highway systems, particularly the simple case of a 1-D platoon of linear vehicles, motivates several recent works, with researchers considering diverse alternatives to achieve coordinated movement of a string of vehicles (see [5], [6] and the references therein).

A simple control strategy is to equip every member of the formation with a compensator that stabilizes its position in closed loop, using as a reference the position of its predecessor on the string plus a desired inter-vehicle spacing. Using integral feedback control, this approach achieves a tight formation in steady state for any constant speed of the leader vehicle. More elaborate approaches also consider using the states of the lead vehicle and/or the states of other members of the formation (see for example [7], [8], [9] and the references therein).

Among the important properties of a formation control architecture we have the stability of the full interconnected system. In [10], the authors discuss the effect of the information flow in vehicle formations and how the eigenvalues of the graph Laplacian matrix play an important role when

This project is funded by the Higher Education Authority (HEA). The programme for research in Third level Institutions Cycle 5 is Co-funded by the Irish Government and the European Union under Ireland's EU Structural Funds Programme 2007- 2013. This work has been (partially) funded by the Advanced Center for Electrical and Electronic Engineering, AC3E, Basal Project FB0008, and by the grant FONDECYT 3160738, CONICYT Chile.

${ }^{1}$ Andrés Peters is with the Advanced Center for Electrical and Electronic Engineering, Universidad Técnica Federico Santa María, Valparaíso, Chile andres.peters@usm. cl.

${ }^{2}$ Oliver Mason is with the Department of Mathematics and Statistics/Hamilton Institute, Maynooth University, Co. Kildare, Ireland and Lero, The Irish Software Research Centre. oliver.mason@nuim.ie.

${ }^{3}$ Richard Middleton is with the Centre for Complex Dynamic Systems and Control, The University of Newcastle, Callaghan NSW 2308, Australia richard.middleton@newcastle.edu.au. determining stability. Other aspects of importance in the architecture are those of performance and safety. Works such as [5], [11], [12] describe how in interconnections that are stable, disturbances may be amplified along the formation resulting in poor performance or even collisions (string instability).

Most strategies also aim to obtain a tight formation, where vehicles maintain a constant inter-vehicle spacing when travelling at a constant speed. This feature is usually a consequence of using certain configurations between the members of the platoon. The motivation behind this is the possibility of reducing fuel consumption by decreasing drag in real applications (See for example [13] and the references therein).

The present work deals with a leader following unidirectional control architecture where the leader moves independently; in such scenarios a follower does not detect or manoeuvre in response to disturbances that affect members behind it. Moreover, the main goal of the architecture is to achieve constant inter-vehicle spacings (with no transients) whenever possible. The main contribution of this paper is to provide a design procedure for a control architecture achieving this tightness property with almost no restrictions on the vehicles and local controllers.

The paper is organized as follows. Section II gives some notation and describes the leader following formation control scheme. Section III presents the associated dynamics obtained by the use of the chosen architecture. In Section IV we present the design choices that provide tightness to the platoon. Some numerical examples and comments on the main results are shown in section V. Section VI contains some conclusions and lines of future work.

\section{FRAMEWORK AND PROBLEM FORMULATION}

\section{A. Notation}

The notation used in this paper follows much of the standard systems and control literature. Lowercase is used for real scalar signals, $x: \mathbb{R} \rightarrow \mathbb{R}$ with specific values of the signal denoted by $x(t)$. Uppercase is used for scalar complexvalued Laplace transforms of signals and transfer functions, $X: \mathbb{C} \rightarrow \mathbb{C}$ with specific values denoted by $X(s)$. For the sake of brevity in the notation, where there is no confusion, the argument $(s)$ will be omitted. Vectors will be denoted as $\underline{x}(t) \in \mathbb{R}^{n}$ and $\underline{X} \in \mathbb{C}^{n}$, while $\underline{x}(t)^{\top}$ and $\underline{X}^{\top}$ denote their transposes. The imaginary unit is denoted by $j$, with $j^{2}=-1$. Boldface will be used for matrices $\boldsymbol{G} \in \mathbb{C}^{n \times m}$ and the $(i, k)$-th entry of $\boldsymbol{G}$ is denoted by $G_{i, k}$. The magnitude of $X$ when $s=j \omega, \omega \in \mathbb{R}$, is denoted by $|X|$ and its 




Fig. 1. Small vehicle platoon. $x_{i}$ : position of the $i$-th vehicle. $e_{i}=x_{i-1}-$ $x_{i}$ : inter-vehicle spacing between the $(i-1)$-th and $i$-th vehicles.

magnitude peak over all possible values of $\omega$ is denoted as $\|X\|_{\infty}:=\sup _{\omega}|X(j \omega)|$.

\section{B. Vehicle models and control strategy}

We consider a platoon of $N \in \mathbb{N}$ vehicles, with positions $x_{i}(t), 1 \leq i \leq N$ modeled by linear time invariant systems (See Figure 1). In the frequency domain, the model of each member of the platoon is given by (omitting initial conditions)

$$
X_{i}=H_{i}\left(U_{i}+D_{i}\right) \quad \text { for } 1 \leq i \leq N,
$$

where $X_{i}$ denotes the Laplace transform of $x_{i}(t), U_{i}$ is the control action and $D_{i}$ is an input disturbance. $U_{i}$ and $D_{i}$ are both acting on the $i$-th member. The transfer functions $H_{i}$ have a single pole at the origin and are strictly proper. Now, we denote the separation errors, for $i>1$, as

$$
\begin{gathered}
e_{i}^{p r e}(t)=x_{i-1}(t)-x_{i}(t)-\varepsilon_{i}, \\
e_{i}^{l e a}=x_{1}-x_{i}-\sum_{j=2}^{i} \varepsilon_{i},
\end{gathered}
$$

and using the Laplace transform,

$$
\begin{aligned}
& E_{i}^{p r e}=X_{i-1}-X_{i}-\frac{\varepsilon_{i}}{s}, \\
& E_{i}^{l e a}=X_{1}-X_{i}-\sum_{j=2}^{i} \frac{\varepsilon_{i}}{s},
\end{aligned}
$$

where the $\varepsilon_{i}>0$ are desired inter-vehicle spacing constants. The error $E_{i}^{\text {pre }}$ is the inter-vehicle spacing between the $i$-th member of the platoon and its predecessor, while the $E_{i}^{\text {lea }}$ is the spacing between the $i$-th member and the leader.

Remark: For simplicity in the exposition we will assume that $\varepsilon_{i}$, and the initial conditions $x_{i}(0)$ and $\dot{x}_{i}(0)$ for $i=$ $1, \ldots, N$ are compatible, that is, such that $e_{i}(0)=0$ for $i=1, \ldots, N$. In this way, we only study the effect of the disturbances $D_{i}$ on the separation errors $E_{i}^{\text {pre }}$. In particular, and without loss of generality, we will set $\varepsilon_{i}=0$ for all $i$ and set every initial condition to zero.

Now we define the control actions

$$
\begin{aligned}
& U_{2}=C_{2} E_{2}^{p r e} \\
& U_{i}=C_{i}\left(\eta_{i} E_{i}^{\text {pre }}+\left(1-\eta_{i}\right) E_{i}^{\text {lea }}\right),
\end{aligned}
$$

where $U_{i}$ is the output of the compensator $C_{i}$ at the $i$-th car and $\eta_{i}$ are stable transfer functions to be designed for $i>2$. With these selections, every member of the string aims to track the position of the leader, while maintaining a safe distance with respect to its immediate predecessor.

In typical leader following settings the weights $\eta_{i}$ are such that $\eta_{i}=\eta$ for all $i$ with $\eta \in(0,1)$. The standard reason for this selection is to achieve a bounded propagation of disturbances along the string (string stability, see for example [5], [14]). In the following sections we will consider $\eta_{i}$ that are dynamic filters instead of real constants.

\section{DYNAMICS OF THE INTERCONNECTED SYSTEM: HOMOGENEOUS CASE}

First, we will consider the homogeneous case, that is, the case where every vehicle has the same model and controller. In other words $H_{i}=H$ and $C_{i}=C$ for all $i=1, \ldots, N$. In this case, the interconnection defined by (6) can be described by the transfer function from the disturbances $\underline{D}=\left[\begin{array}{lll}D_{1} & \cdots & D_{N}\end{array}\right]^{\top}$ to the positions $\underline{X}=\left[\begin{array}{lll}X_{1} & \cdots & X_{N}\end{array}\right]^{\top}$ :

$$
\underline{X}=(\boldsymbol{I}-H C \boldsymbol{G})^{-1} H \underline{D},
$$

where $\boldsymbol{I}$ is the $N \times N$ identity matrix and $\boldsymbol{G}$ is the matrix:

$$
\boldsymbol{G}=\left[\begin{array}{ccccc}
0 & & & & \\
1 & -1 & & & \\
1-\eta_{3} & \eta_{3} & -1 & & \\
\vdots & & \ddots & \ddots & \\
1-\eta_{N} & & \ldots & \eta_{N} & -1
\end{array}\right]
$$

The term $G_{1,1}=0$ implies that the first vehicle moves independently and its position is given by

$$
X_{1}=H D_{1}
$$

Although we have homogeneous vehicles, we consider $\eta_{i}$ to be dynamic and arbitrary for $i>2$. If we define the usual complementary sensitivity functions as

$$
\begin{aligned}
& T=\frac{H C}{1+H C}, \\
& S=1-T,
\end{aligned}
$$

we have

$$
\underline{X}=\left[\begin{array}{ccccc}
S & & & & \\
-T & 1 & & & \\
\left(\eta_{3}-1\right) T & -\eta_{3} T & 1 & & \\
\vdots & & \ddots & \ddots & \\
\left(\eta_{N}-1\right) T & & & -\eta_{N} T & 1
\end{array}\right]^{-1} S H \underline{D} .
$$

Since the matrix to be inverted is lower triangular, we can easily express the dynamics of the vehicle positions.

Remark: In the following we will focus on the effect of the disturbance on the first vehicle $D_{1}$ and assume that for all $i>1, D_{i}=0$ for all $s$. The reason for this is that we are mainly interested in the behaviour of the string in nominal conditions, that is, when the movement of the string is only caused by the movement of the leader. At the same time, we will obtain conditions on the $\eta_{i}$ based on this assumption. 
We have that

$$
\underline{X}=\left[\begin{array}{c}
1 \\
T \\
\tilde{T}_{3} \\
\tilde{T}_{4} \\
\vdots \\
\tilde{T}_{N}
\end{array}\right] H D_{1}
$$

where

$$
\tilde{T}_{k}=T-\sum_{i=3}^{k}\left(T^{k-i+1} S \prod_{j=i}^{k} \eta_{j}\right)
$$

for $k=3, \ldots, N$.

Now, we are interested in the spacing errors from one vehicle to its immediate predecessor, i.e. $E_{k}^{p r e}=X_{k-1}-X_{k}$ for $k=2, \ldots, N$. Then these errors are given by

$$
\begin{aligned}
{\left[\begin{array}{c}
E_{2}^{p r e} \\
\vdots \\
E_{N}^{p r e}
\end{array}\right] } & =\left[\begin{array}{cccc}
1 & -1 & & \\
& \ddots & \ddots & \\
& & 1 & -1
\end{array}\right] \underline{X} \\
& =\left[\begin{array}{c}
S \\
T-\tilde{T}_{3} \\
\tilde{T}_{3}-\tilde{T}_{4} \\
\vdots \\
\tilde{T}_{N-1}-\tilde{T}_{N}
\end{array}\right] H D_{1} .
\end{aligned}
$$

We can rewrite the right hand side as

$$
\left[\begin{array}{c}
S \\
T-\tilde{T}_{3} \\
\tilde{T}_{3}-\tilde{T}_{4} \\
\vdots \\
\tilde{T}_{N-1}-\tilde{T}_{N}
\end{array}\right] H D_{1}=\left[\begin{array}{c}
1 \\
\mathcal{T}_{3} \\
\vdots \\
\mathcal{T}_{N}
\end{array}\right] S H D_{1}
$$

where $\mathcal{T}_{k}$ satisfies

$$
\mathcal{T}_{k}=\eta_{k} T+\left(\eta_{k} T-1\right) \sum_{i=3}^{k-1}\left(T^{k-i} \prod_{j=i}^{k-1} \eta_{j}\right)
$$

for $k=3, \ldots, N-1$, with $\mathcal{T}_{3}=\eta_{3} T$. If $\eta_{3}=\eta_{4}=\ldots=$ $\eta_{N}=\tilde{\eta}$, then $\mathcal{T}_{k}=(\tilde{\eta} T)^{k-1}$. This is in agreement with results presented in [5].

\section{A. Filters $\eta_{i}$ selections for homogeneous case}

Now, we study a possible selection for the sequence $\left\{\eta_{k}\right\}$. From a practical point of view (for example optimization of fuel consumption [13]) it would be ideal to have $E_{i}^{\text {pre }}=0$ for all $s$ and all $i \geq 2$. However, the inter-vehicle spacings between the first and second vehicles $E_{2}^{\text {pre }}=S H D_{1}$ and between the second and third vehicles $E_{3}^{\text {pre }}=\eta_{3} T S H D_{1}$ are fixed for any possible selection of $\eta_{k}$ or controllers. Nevertheless, for $k=4$ we have

$$
\mathcal{T}_{4}=\eta_{4} T+\left(\eta_{4} T-1\right) T \eta_{3},
$$

and solving $\mathcal{T}_{4}=0$ yields

$$
\eta_{4}=\frac{\eta_{3}}{1+\eta_{3} T}
$$

For $i=5$ we have

$$
\begin{aligned}
\mathcal{T}_{5} & =\eta_{5} T+\left(\eta_{5} T-1\right)\left(\eta_{3} \eta_{4} T^{2}+\eta_{4} T\right) \\
& =\eta_{5} T+\left(\eta_{5} T-1\right) \eta_{4} T\left(1+\eta_{3} T\right)
\end{aligned}
$$

and using $\eta_{4}$ obtained in (20) we have

$$
\mathcal{T}_{5}=\eta_{5} T+\left(\eta_{5} T-1\right) \eta_{3} T .
$$

Solving $\mathcal{T}_{5}=0$ now yields

$$
\eta_{5}=\frac{\eta_{3}}{1+\eta_{3} T}
$$

It is straightforward to compute that for a fixed $\eta_{3}$, the selection

$$
\eta_{k}=\frac{\eta_{3}}{1+\eta_{3} T}, \quad k \geq 4
$$

yields $\mathcal{T}_{k}=0$ for all $s$.

For movements of the leader, this selection allows a response of the vehicle platoon that resembles the movement of a train (with the exception of the first two followers). It must be noted that the selection of $\eta_{3}$ can be any stable transfer function and it must be selected in order that $\eta_{3} /\left(1+\eta_{3} T\right)$ is also stable.

The selection of the sequence $\left\{\eta_{k}\right\}$ was made with the aim of obtaining $E_{k}^{\text {pre }}=0$ for all $s$ whenever possible for disturbances (or movement) at the leader. These selections may not provide a satisfactory response of the vehicle string for disturbances at the followers. This is currently being investigated in detail with aims to assess the string stability of the interconnection. Nevertheless, in Section $\mathrm{V}$ we consider the effect of disturbances at followers and the response of the interconnection in numerical simulations.

\section{DYNAMICS OF THE INTERCONNECTED SYSTEM: HeTEROGENEOUS CASE}

Now we consider the heterogeneous case. We will use an alternative method for obtaining a sequence $\eta_{k}$ that achieves a tight formation. First, we define

$$
T_{k}=\frac{H_{k} C_{k}}{1+H_{k} C_{k}} .
$$

In general, for $D_{k}=0$ for $k>1$, the vehicle positions are given by (omitting initial conditions)

$$
\begin{aligned}
& X_{1}=H_{1} D_{1}, \\
& X_{2}=T_{2} X_{1}, \\
& X_{k}=T_{k}\left(\left(1-\eta_{k}\right) X_{1}+\eta_{k} X_{k-1}\right), \quad k>2 .
\end{aligned}
$$

Since it is not possible, as in the homogeneous case, to achieve $E_{3}^{\text {pre }}=0$ we fix $\eta_{3}$ to be arbitrary and search for 


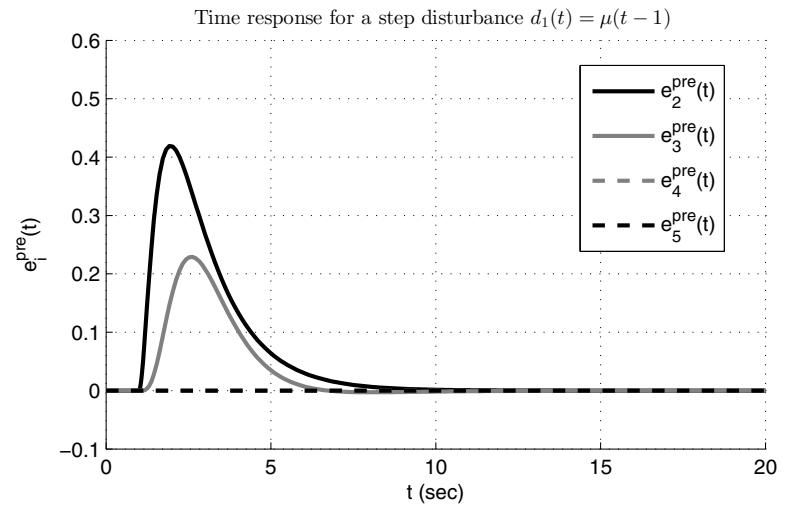

Fig. 2. Time response of the inter-vehicle spacings with a step disturbance at the leader $d_{1}(t)=\mu(t-1)$ for a platoon with non-homogeneous weights $\eta_{k}$.

$\eta_{k}$ such that $E_{k}^{\text {pre }}(s)=0$ for all $s$ with $k>3$. In particular we have that

$$
\begin{aligned}
E_{3}^{\text {pre }} & =X_{2}-X_{3}=T_{2} X_{1}-T_{3}\left(\left(1-\eta_{3}\right) X_{1}+\eta_{3} X_{2}\right) \\
& =\left(T_{2}-T_{3}\left(1-\eta_{3}\right)-\eta_{3} T_{2} T_{3}\right) X_{1}
\end{aligned}
$$

and we set

$$
\tilde{T}=T_{3}\left(1-\eta_{3}+\eta_{3} T_{2}\right) .
$$

By setting $E_{k}^{p r e}(s)=0$ in the control signal definition in (6) we obtain

$$
X_{k}=\frac{H_{k} C_{k}\left(1-\eta_{k}\right)}{1+H_{k} C_{k}\left(1-\eta_{k}\right)} X_{1}, \quad k>3 .
$$

Now, for $E_{4}^{\text {pre }}$ we have

$$
E_{4}^{p r e}=X_{4}-X_{3}=\frac{H_{4} C_{4}\left(1-\eta_{4}\right)}{1+H_{4} C_{4}\left(1-\eta_{4}\right)} X_{1}-\tilde{T} X_{1},
$$

and setting $E_{4}^{\text {pre }}=0$ yields

$$
\frac{H_{4} C_{4}\left(1-\eta_{4}\right)}{1+H_{4} C_{4}\left(1-\eta_{4}\right)}=\tilde{T} .
$$

Working iteratively we can conclude that in order to have $E_{k}^{p r e}=0$ for $k>3$, we must have

$$
1-\eta_{k}=\frac{\tilde{T}}{H_{k} C_{k}(1-\tilde{T})} .
$$

Remarks: The expression in (36) gives a selection of the filters $\eta_{k}$ in order to achieve tight inter-vehicle spacings with no transient when only the leader moves independently (there are no disturbances). These filters for every vehicle depend on the local parameters $H_{k}$ and $C_{k}$ and on the parameter $\tilde{T}$ which is fixed by the selection of $\eta_{3}$ and the second and third vehicles of the string. This in turn means that any vehicle that desires to merge into the platoon just needs to be aware of $\tilde{T}$ on top of the usual requirements for leader following schemes (assuming that every vehicle in front is following the same algorithm for implementing the control).

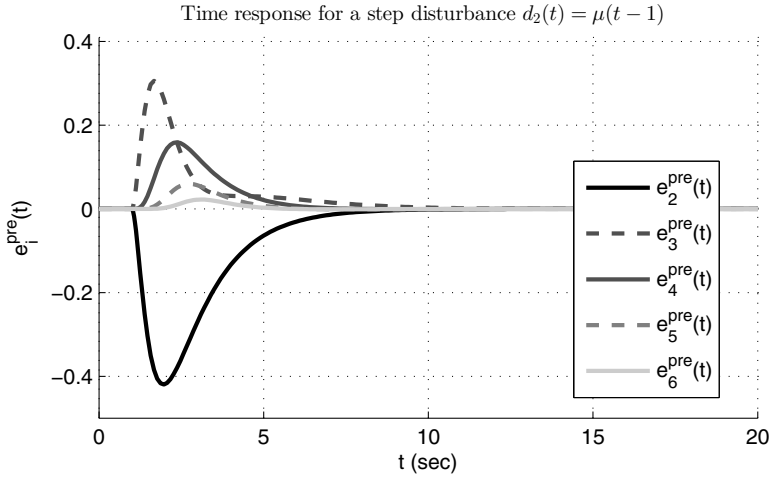

Fig. 3. Time response of the inter-vehicle spacings with a step disturbance at the second member $d_{2}(t)=\mu(t-1)$ for a platoon with non-homogeneous weights $\eta_{k}$.

On an additional note, (36) gives constraints on the local controllers considering that once $\tilde{T}$ is fixed, $C_{k}$ must be chosen such that $\eta_{k}$ is a proper transfer function and the local closed loop at the $k$-th vehicle is stable.

Finally, we note that the outcome of having no transients for the inter-vehicle spacings after the third vehicle is highly sensitive to model uncertainty. This is the topic of ongoing research.

\section{NUMERICAL EXAMPLES}

\section{A. Homogeneous case}

We consider a homogeneous string with the parameters (Taken from [5])

$$
\begin{aligned}
& H=\frac{1}{s(0.1 s+1)}, \\
& C=\frac{2 s+1}{s(0.05 s+1)} .
\end{aligned}
$$

Additionally we set $\eta_{3}=0.5$ and consequently

$$
\begin{aligned}
T & =\frac{400 s+200}{s^{4}+30 s^{3}+200 s^{2}+400 s+200}, \\
\eta_{k} & =\frac{1}{2+T}=\frac{0.5 s^{4}+15 s^{3}+100 s^{2}+200 s+100}{s^{4}+30 s^{3}+200 s^{2}+600 s+300},
\end{aligned}
$$

for $k>3$, which are stable for the particular selection of $C$ and $H$. For a step input to the lead vehicle, $d_{1}(t)=\mu(t-1)$ we obtain the transient response shown in Figure 2. All the inter-vehicle spacings $e_{i}^{\text {pre }}(t)$ are 0 for $i>3$ as desired and imposed in the derivation of the sequence $\eta_{k}$.

As dealing with the expressions for the response of the platoon for disturbances to the followers requires more derivations, we will illustrate this case with a simulation. In Figure 3 we can see the time response of the platoon to a disturbance at the second vehicle $d_{2}(t)=\mu(t-1)$. The inter-vehicle spacings along the string have magnitude peaks that are smaller than the peak for $e_{2}^{p r e}(t)$. This suggests that the sequences of transfer functions from disturbances at the followers to other followers could be string stable. A future extension is then to compute such sequences for arbitrary parameter values, and find the conditions for string stability. 


\section{B. Heterogeneous case}

Now we consider a heterogeneous string. For the first three vehicles we have

$$
\begin{array}{r}
H_{1}=H_{2}=H_{3}=H=\frac{1}{s(0.1 s+1)}, \\
C_{2}=C_{3}=C=\frac{2 s+1}{s(0.05 s+1)} .
\end{array}
$$

For $k=4, \ldots, 8$ we have

$$
H_{k}=\frac{1}{s(0.1 s / k+1)} .
$$

With these parameters and setting $\eta_{3}=0.5$ we have

$$
\begin{aligned}
T_{2}=T_{3} & =T, \\
\tilde{T} & =\frac{T(1+T)}{2},
\end{aligned}
$$

with $T$ obtained in (39) above. A simple numerical check shows that $C_{k}=C$ defined in (41) implies that every $T_{k}$ is stable for $k>3$. Therefore, we have that

$$
\eta_{k}=1-\frac{H(1+T)}{H_{k}(2+T)}, \quad k>3 .
$$

It is possible to check that $\eta_{k}$ is strictly proper for $k>3$. For the same disturbances as in the homogeneous case, the time responses are very similar therefore we omit them.

\section{CONCLUSIONS AND FUTURE WORKS}

In this paper we have considered a unidirectional strategy for the formation control of a string of vehicles. We have shown that the vehicles can use filtered measurements of the leader and their immediate predecessor in order to obtain a quasi train-like behaviour under nominal operation (with no disturbances and starting from the desired formation). This is feasible even for a heterogeneous string, namely, for a string with different vehicles using different controllers.

The most noteworthy feature of the control strategy is that the vehicles do not need to know all the characteristics of every predecessor but only of the first few vehicles. This allows for reduced coordination requirements and the possibility for new vehicles to merge in the platoon in a simplified fashion.

Future lines of work include a more detailed study of the behaviour of the interconnection under disturbances at any member of the platoon. It is also of interest to assess the string stability of the control architecture and the behaviour when there are modelling errors or uncertainties. We also aim to consider disruptions of the leader state that must be transmitted to the followers.

\section{REFERENCES}

[1] W. Levine and M. Athans, "On the optimal error regulation of a string of moving vehicles," Automatic Control, IEEE Transactions on, vol. 11 , no. 3, pp. 355 - 361, jul 1966.

[2] K. C. Chu, "Decentralized control of high-speed vehicular strings," Transportation Science, vol. 8, no. 4, pp. 361-384, 1974.

[3] C. C. Chien and P. Ioannou, "Automatic vehicle-following," in American Control Conference, 1992, june 1992, pp. 1748 -1752.

[4] D. Swaroop and J. Hedrick, "String stability of interconnected systems," Automatic Control, IEEE Transactions on, vol. 41, no. 3, pp 349 -357, mar 1996.

[5] P. Seiler, A. Pant, and K. Hedrick, "Disturbance propagaton in vehicle strings," IEEE Transactions on Automatic Control, vol. 49, no. 10, pp. 1835-1841, 2004.

[6] S. Klinge and R. Middleton, "Time headway requirements for string stability of homogeneous linear unidirectionally connected systems," in Decision and Control, 2009 held jointly with the 2009 28th Chinese Control Conference. CDC/CCC 2009. Proceedings of the 48th IEEE Conference on, dec. 2009, pp. $1992-1997$.

[7] M. R. Jovanovic and B. Bamieh, "On the ill-posedness of certain vehicular platoon control problems," Automatic Control, IEEE Transactions on, vol. 50, no. 9, pp. 1307-1321, 2005.

[8] H. Hao, H. Yin, and Z. Kan, "On the robustness of large 1-d network of double integrator agents," in American Control Conference (ACC), 2012, 2012, pp. 6059-6064.

[9] F. Lin, M. Fardad, and M. R. Jovanovic, "Optimal control of vehicular formations with nearest neighbor interactions," Automatic Control, IEEE Transactions on, vol. 57, no. 9, pp. 2203-2218, 2012.

[10] J. Fax and R. Murray, "Information flow and cooperative control of vehicle formations," Automatic Control, IEEE Transactions on, vol. 49 no. 9, pp. 1465-1476, 2004.

[11] R. Middleton and J. Braslavsky, "String instability in classes of linear time invariant formation control with limited communication range," Automatic Control, IEEE Transactions on, vol. 55, no. 7, pp. 1519 -1530 , july 2010.

[12] P. Barooah and J. P. Hespanha, "Error amplification and disturbance propagation in vehicle strings with decentralized linear control," in Decision and Control, 2005 and 2005 European Control Conference. CDC-ECC'05. 44th IEEE Conference on, 2005, pp. 4964-4969.

[13] C. Bonnet and H. Fritz, "Fuel consumption reduction in a platoon: Experimental results with two electronically coupled trucks at close spacing," SAE Technical Paper, Tech. Rep., 2000.

[14] A. Peters, R. Middleton, and O. Mason, "Leader tracking in homogeneous vehicle platoons with broadcast delays," Automatica, vol. 50, no. 1, pp. 64-74, 2014 